\title{
Innovative Congestion Control System for Ambulance using RF
}

\author{
Ch. Ramaiah ${ }^{1}$ \\ Asst. Prof, \\ Department of Electrical \& Electronics Engineering \\ Vignan institute of Technology and Science \\ Telangana.
}

\author{
M. Harika², Ch. Arun ${ }^{3}$, M. Shivasai Reddy ${ }^{4}$ \\ U.G Student, \\ Department of Electrical \& Electronics Engineering \\ Vignan Institute of Technology and Science \\ Telangana.
}

\begin{abstract}
Congestion problem is a fact which contributed large impact on the transport system in country. This cause many problems especially when there are emergency cases at traffic light junctions which are always busy with so many vehicles. A congestion control system is designed in order to solve these problems. This system was designed in such a way that it should be activated when it received signal from ambulance based on radio frequency (RF) transmission and used microcontroller to change the sequence back to the normal sequence before the emergency mode was activated. This system will lessen accidents which often happen at the traffic light intersections because of other vehicles had to gather together for giving a special route to emergency vehicle. As the result, this project is successful and implementing the wireless communication i.e. the radio frequency (RF) transmission in the traffic light control system for emergency vehicles like ambulance. The main aim of this project is to design a system which is capable of identifying the emergency situation in ambulance and automatically controls the traffic at the signal. In future, this system can be improved by controlling real traffic situation, in fact improving present traffic light system technology.
\end{abstract}

Keywords: RF, Arduino nano

\section{INTRODUCTION}

The existing traffic control system has a lot of disadvantage because of its fixed time method used. The traffic signal will not change based on the real time traffic on road near the junction of two or more roads. Due to this the traffic congestion cannot be controlled efficiently and the utilization of road cannot be done to its maximum limit[4]. In country like India, day by day, the no of vehicles on road is increasing due to which congestion is a major problem. Traffic congestion leads to long waiting time, fuel loss and also the money wastage. Congestion results in pollution which in turn affects the living. In India traffic is non-lane based and chaotic, so congestion control provided should be better. On account of this congestion it is difficult for the emergency vehicles like ambulance and fire brigade to reach its destination on time which may cost the lives of people. To overcome these problems traffic control system are made efficient with the use new technologies like ZigBee, RF and GSM. Over the other technologies RF has advantage because it is cost efficient system an there will be no interruption in communication even in bad weather conditions . RF is an Radio Frequency which is a wireless technology.
The traffic light system designed by Levi L. Rose [1] used only for ambulance. Sensor is used to transmit signal that has been installed in every ambulance to the receiver which has been placed at every traffic light junction. When ambulance reaches the traffic light junction, the signal code will sent information of frequency modulation to the receiver. The receiver demodulates the code that had received and the red traffic light will activate at all the junctions. Thus, ambulance will have special route to reach the target.

The traffic light system by M. R. Smith et al [2] provided strike one of the approaching an ambulance to find a way out from traffic congestion and lead the ambulance to the target. The ambulance also may take control of traffic light at an junction. A transmitter placed on an ambulance transmits a signal to the receivers positioned at the traffic lights whenever it is on emergency mode. The received signal is then prepared by a master controller which in turn assume the sequence of the traffic light to control the traffic flow at the junction which taken by the emergency vehicle. The master controller also provides an output which display signs to indicate that there is an emergency vehicle to the other road users from other direction at the traffic light junction. Whether the ambulance has passed through the junction or not is indicated by the display system.

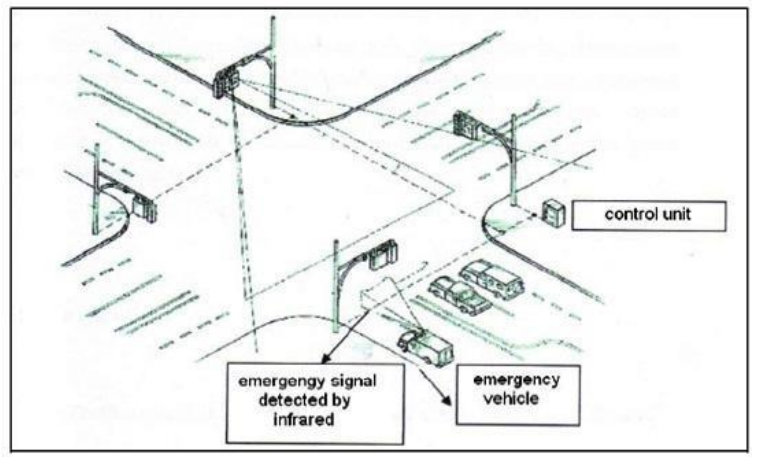

Fig.1 Four-traffic light intersection traffic light

W. L. Mitchell has designed a traffic light control system [3] which had get control of the traffic congestion problem and provided an emergency path for the ambulance where the radio transmitter and antenna placed on the ambulance. The radio will transmit the signal to the other vehicle that closeby. The radio receiver had placed at four junction traffic light will receive the emergency signal from ambulance that passed by the junction. The first signal code 
contains a frequency for emergency vehicle while the second signal code contains a frequency for another vehicle. The transmitted signals provide different traffic light pole in normal condition or emergency. When the receiver received the signal from ambulance transmitter, traffic light system for ambulance will be activated.

A traffic light control system designed by Carl J. Obeck [4] consists of two-way communication among emergency vehicles approaching a busy intersection with one or more traffic lights. The system temporarily prevent the series of the traffic light and provides the most successful method of routing the vehicle through the junction while redirecting general traffic. As part of the invention, the traffic light control system will inform the ambulance which it has received the transmitted signal. The stored definite traffic patterns may in one representation is responsive to manual intervention from a dispatching centre or to time-of-day conditions. The traffic light control equipment may be operated under control of data or voice transmitted from the emergency vehicle's regular two-way voice communications system to a central control station.

According to all these papers, a convenient wireless communication among emergency vehicles and the traffic light is by using RF. The design of this project is using the radio frequency of $434 \mathrm{MHz}$ compared to the range of about $3 \mathrm{kHz}$ to $300 \mathrm{GHz}$ of frequency which have been reserved for the RF theoretically. There are three objectives to be achieved in this project. First is to analyze and implement wireless communication; Radio Frequency (RF) transmission system in traffic light control system used for the emergency vehicles. Second is to design a traffic light sequence for emergency mode when receive signal from emergency vehicle like ambulance. Last objective is to change the sequence back to the normal sequence before the emergency mode was activated. This project has assisted in implementing the wireless communication by using the radio frequency $(\mathrm{RF})$ transmission of $434 \mathrm{MHZ}$ in the traffic light control system.

\section{PRINCIPLE}

The RF transmission system employs with receiver/transmitter $(\mathrm{Rx} / \mathrm{Tx})$ pair operating at 434 $\mathrm{MHz}$. The transmitter module takes serial input and then transmits these signals through $\mathrm{RF}$. The receiver receives the transmitted signals and then sends to the module placed away from the source of transmission.

A radio frequency (RF) signal starts as an electrical alternating current (AC) signal that is originally generated by a transmitter [6]. This AC signal is sent through a copper conductor which usually a coaxial cable and radiated out of an antenna element in the form of an electromagnetic wave. The Change of current flow in the antenna produce changes in the electromagnetic fields around the antenna.

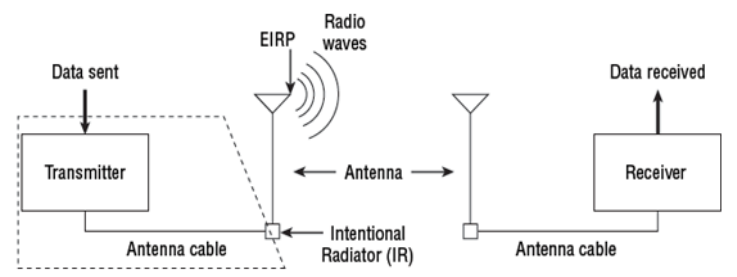

Fig.2 The RF module with TX and RX

\section{METHODOLOGY}

RFR

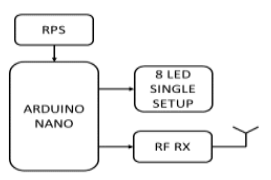

RFTX

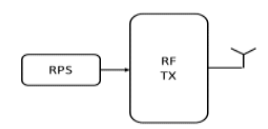

Fig.3 Project block diagram

Arduino Nano: Arduino nano has same functionalities as Arduino Duemilanove but with a different package. The Nano is inbuilt With the ATmega328P microcontroller, similar to that of Arduino UNO. The main difference between the two microcontroller is that the UNO board is present in PDIP (Plastic Dual-In-line Package) form with 30 pins and Nano is available in TQFP (plast quad flat pack) with 32 pins. The extra 2 pins of Arduino Nano served for the ADC features, while UNO has 6 ADC ports but Nano has 8 ADC ports. The Nano board doesnot have a DC power jack as another Arduino boards, but instead has a mini-USB port. This port is used not only for programming but also to serial monitoring. The fascinating function of Nano is that it will choose the well builtt power source with its potential difference, and the power source selecting jumper is invalid.

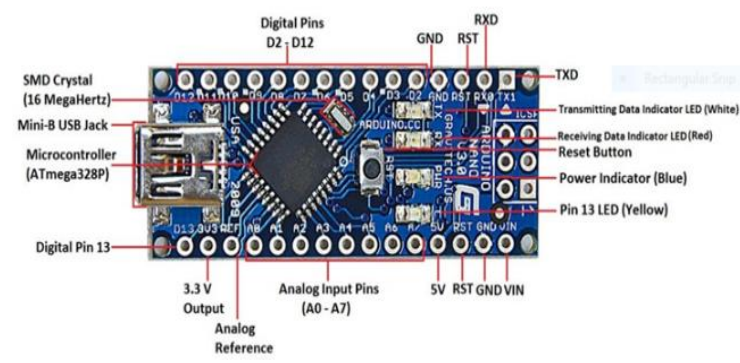

Fig. 4 Arduino nano(microcontroller)

LED: A light-emitting

a semiconductor light path that emits light when current flows through it. Electrons in the semiconductor merge with electron holes, releasing energy in the form of photons. The colour of the light (corresponding to the energy of the photons) is decided by the energy required for electrons to cross the band gap of the semiconductor. White light is not only obtained by using multiple semiconductors but also by a layer of light-emitting phosphor on the semiconductor device

RF Transmitter: The STT-433 is absolute for remote control applications where low cost and longer range is required. The transmitter operates from a1.5-12V supply, making it 
perfect for battery-powered applications. The transmitter employs a stabilized oscillator, ensuring accurate frequency control for better range performance. Output power and harmonic emissions are very easy to control, making FCC and ETSI compliance easy. The manufacturing-friendly SIP style package and low-cost make the STT-433 appropriate for high volume applications.
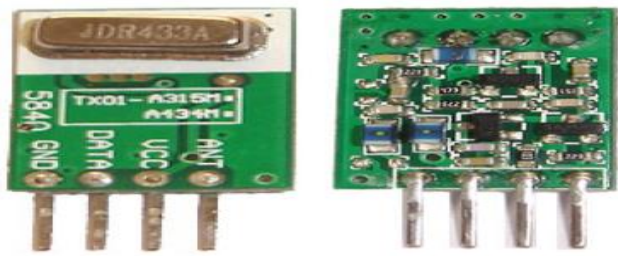

Fig. 5 RF Transmitter

RF receiver: The STR-433 is ideal for short-range remote control applications where cost is a primary anxiety. The receiver module doesn't required external RF components except for the antenna. It generates almost no emissions, making FCC and ETSI approvals easy. The superregenerative design shows exceptional sensitivity at a very low cost. The manufacturing-friendly SIP style package and low-cost make the STR-433 suitable for high range volume applications.

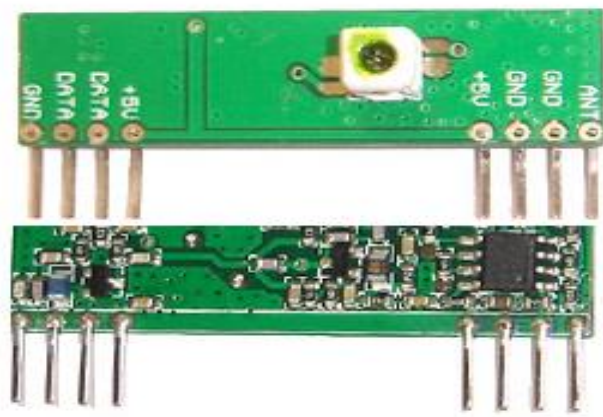

Fig. 6 RF Receiver

\section{HARDWARE REQUIREMENT}

1. Arduino nano

2. RPS

3. 8 LED

4. RF RX

5. RF TX

\section{SOFTWARE REQUIREMENTS}

1. Arduino IDE

2. Embedded

\section{RESULT}

1. Cost effective

2. Less prone to error

3. No need of traffic police.

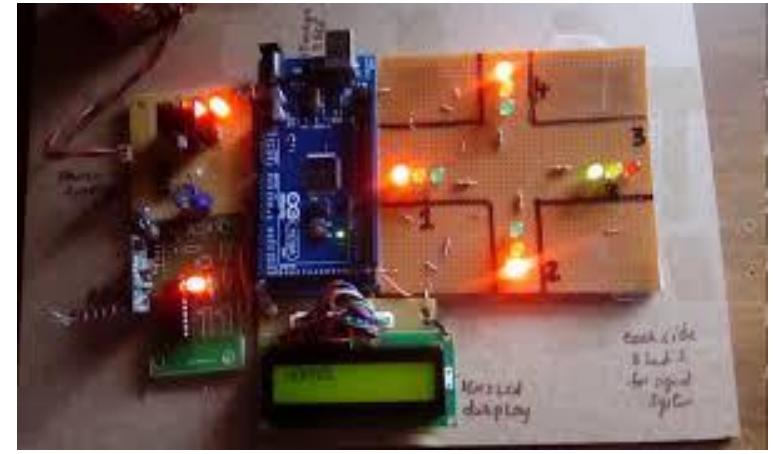

Fig. 7 Arduino based congestion control system for ambulance using RF

The Emergency Mode Sequence:

The emergency mode is activated when the RF receiver received the transmitted signal from the RF transmitter to override the normal sequence of the traffic light. For example, an ambulance arrives at the traffic light named 4 and the green light of the traffic light named 1 is on.

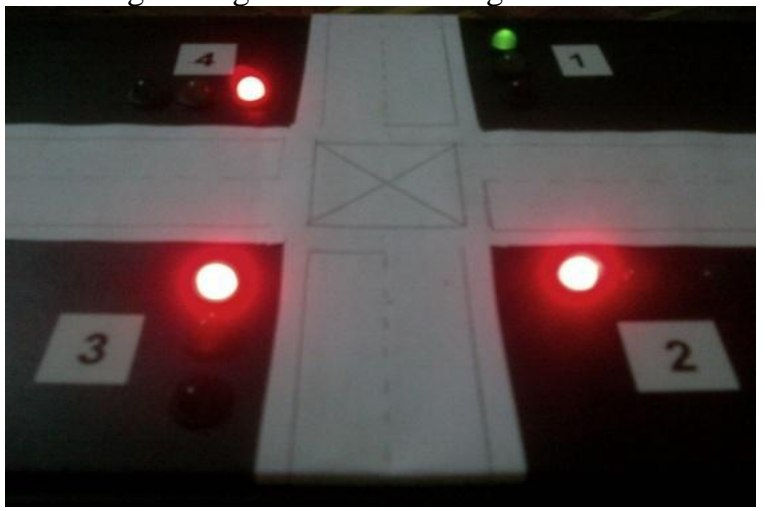

Fig. 15 The green light of traffic light named 1 is on

When the push-on button no. 4 is turned on in the ambulance by the driver, the $\mathrm{RF}$ receiver received the transmitted signal and changed the sequence to the emergency sequence mode. The emergency sequence mode started when the yellow of traffic light named 1 is on for 2 seconds. Then the green of traffic light named 4 is on for 10 seconds and then the yellow light of the same traffic light is turned on for 2 seconds.

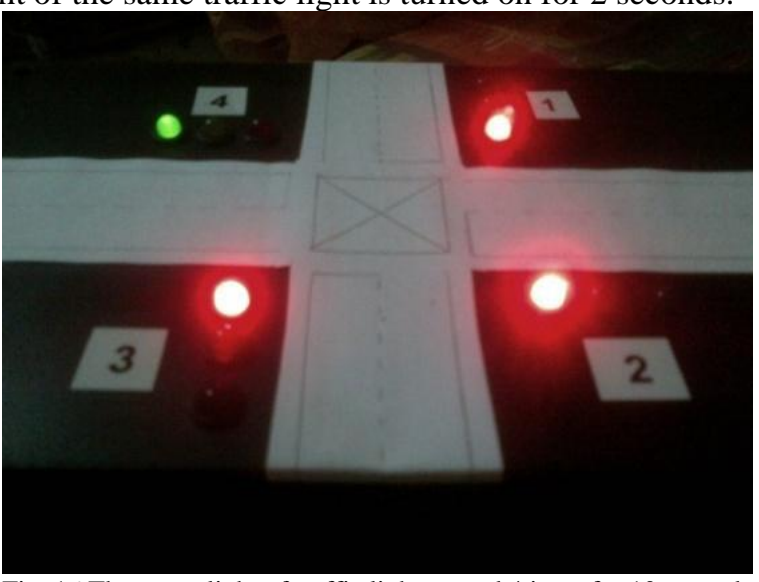

Fig. 16 The green light of traffic light named 4 is on for 10 seconds 


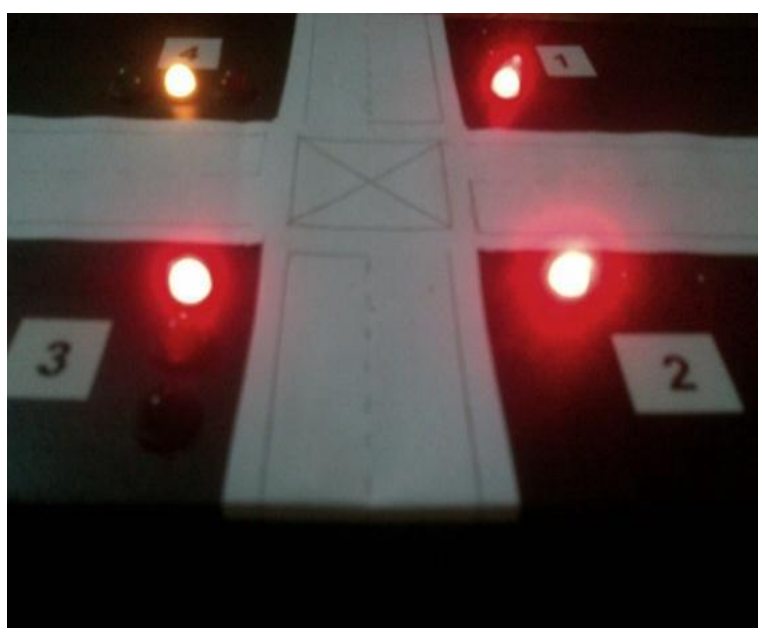

Fig.17 The yellow light of traffic light named 4 is on for 2 seconds

The emergency sequence mode is ended when the sequence of the traffic light is back to the normal sequence which the green light of traffic light named 1 is turned back on for the left time before the emergency sequence mode is activated.

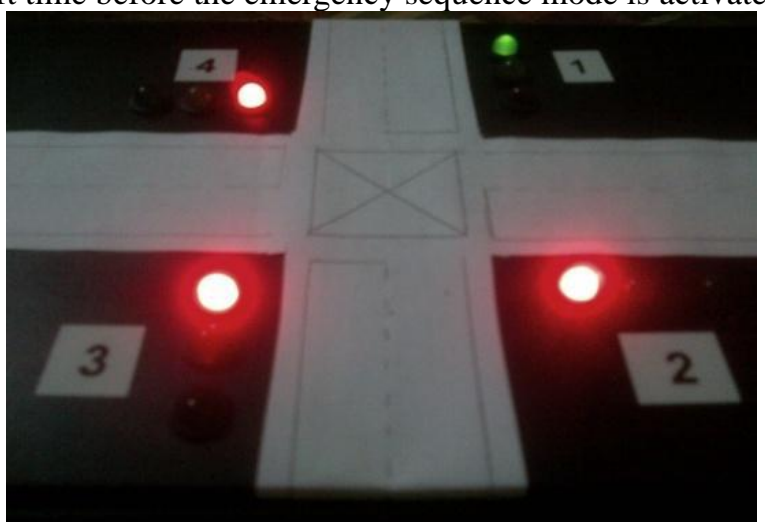

Fig. 18 The sequence of the traffic light is back to original position

\section{CONCLUSION}

As a conclusion, this project have achieved the main objective stated earlier which is analyzing and exploring the wireless communication; the radio frequency (RF) transmission in the traffic light control system for emergency vehicles. The model of this project is using the frequency of $434 \mathrm{MHz}$ compared to the range of about $3 \mathrm{kHz}$ to $300 \mathrm{GHz}$ of frequency which have been reserved for the RF theoretically. Besides, the performance of this project proved that the other objectives have been successfully attained which are designing an emergency sequence mode of traffic light when emergency vehicles passing by an junction and changing the sequence back to the normal sequence before the emergency mode was activated. The sequences for this project have been developed using the programming in the microcontroller. In future, this prototype system can be upgraded by controlling the real traffic situation and the study can be done by investigating the length and transmission issue for the system can be operated with this traffic light control system.

\section{ACKNOWLEDGEMENTS}

We are grateful to Vignan Institute of technology and Science(VITS) for their kind help for supplying the electronic components and giving their laboratory facility to complete this study.

\section{REFERENCES}

[1] Levi L. Rose, "Emergency Traffic Control System with Security Transmission Coding”, United States Patent, April 5th, 1997.

[2] Paul J. Davidson and Henry L. Pfister, "Emergency Vehicle Warning and Traffic Control System", United States Patent, October 4th, 1998.

[3] Willbur L. Mitchell, "Traffic Light Control For Emergency Vehicles", United States Patent, April 17th, 1994.

[4] William E. Brill, "Emergency Vehicle Detection System", United States Patent, March 26th, 2002.

[5] Carl J. Obeck, "Traffic Signal Control For Emergency Vehicles", United States Patent, May 7th, 1998.

[6] David D. Coleman, David A. Westcott, "CWNA: Certified Wireless Network Administrator Official Study Guide", Sybex, April 06, 2009.

[7] "RF Based Wireless Remote using Tx-Rx Modules", Chawla Radios and Electricals, August 11th, 2011.

[8] http://www.blurtit.com/q335716.html, What Is The Difference Between Decoder And Encoder? Lukas Hoffmann, "PIC 16F877A Tutorials for Pitt Robotics Club", 2010. 